\title{
Uso de los servicios de salud y progresión al sida entre personas con infección por VIH en Belo Horizonte (Minas Gerais), Brasil
}

\author{
Francisco de Assis Acurcio ${ }^{1}$ y Mark Drew Crosland Guimarães ${ }^{2}$
}

\begin{abstract}
RESUMEN Se realizó un estudio prospectivo no concurrente para investigar la relación entre la progresión al sida de personas diagnosticadas de infección por el virus de la inmunodeficiencia humana (VIH) y variables sociodemográficas, de comportamiento y, principalmente, de dificultad en el acceso a los servicios públicos de salud de remisión para pacientes con VIH/sida. El curso de la infección fue monitoreado desde la primera hasta la última visita a los servicios de salud por medio de una clasificación individual basada en el diagnóstico, de acuerdo con las categorías clínicas establecidas en 1993 por los Centros para el Control y la Prevención de Enfermedades de los Estados Unidos de América. Participaron en el estudio 758 pacientes recibidos entre 1989 y 1992 en los servicios públicos de remisión para pacientes de sida de la ciudad de Belo Horizonte, Minas Gerais. Todos habian sido diagnosticados de infección por VIH y clasificados en estadios anteriores al sida. Tanto los pacientes que evolucionaron al sida durante el estudio como los que no lo hicieron fueron comparados en cuanto a las características de exposición seleccionadas.

Durante el período de estudio, 39,5\% de los pacientes progresaron al sida. La mediana del tiempo sin sida de todos los pacientes fue de 32,4 meses. El análisis multivariado mostró que en los pacientes que tuvieron menos de 8,8 consultas al año ( $R R=0,36 ;$ IC95\%: 0,26 a 0,50) y por lo menos un intervalo mayor de 6 meses entre consultas ( $\mathrm{RR}=0,37$; IC95\%: 0,25 a $0,55)$, el riesgo de desarrollar el sida fue menor que en los demás. El riesgo fue mayor en pacientes con edad $\geq 30$ años ( $\mathrm{RR}=1,37 ;$ IC95\%: 1,03 a 1,84), en los que no recibían tratamiento con azidovudina (AZT) (RR = 1,91; IC95\%: 1,37 a 2,64) y en los que fueron clasificados en la categoría " $B$ " como estadio inicial ( $\mathrm{RR}=4,83$; IC95\%: 3,59 a 6,48) de la enfermedad. Los resultados de esta investigación serán útiles en la planificación y organización de servicios de atención para personas con VIH, ya que revelan la dinámica de la oferta y demanda de servicios por esa clientela. Se recomienda dar prioridad a la intervención temprana con énfasis en la atención ambulatoria continua y estudiar más ampliamente el proceso de búsqueda y obtención de cuidados de salud por parte de personas con infección por VIH.
\end{abstract}

1 Universidad Federal de Minas Gerais, Brasil, Escuela de Veterinaria, Departamento de Farmacia Social, Facultad de Farmacia.

2 Universidad Federal de Minas Gerais, Brasil, Departamento de Medicina Preventiva y Social. Facultad de Medicina. Los pedidos de separatas y otra correspondencia deben enviarse a este autor, a la siguiente dirección: Av. Alfredo Balena, 190, $10^{\circ}$ andar, Caixa postal 340, CEP 30.130-100, Belo Horizonte, Minas Gerais, Brasil.
Desde que se identificaron los primeros casos de sida en el Brasil a principios de los años ochenta, la epidemia causada por el virus de la inmunodeficiencia humana $(\mathrm{VIH})$ ha ocupado un lugar cada vez más destacado entre los principales problemas de salud pública del país. Esto se debe a tres razones: 1) el temor que generan las características de un síndrome que todavía es universalmente incurable, a pesar de los importantes avances logrados recientemente en su conocimiento y tratamiento; 2) la magnitud del problema: 
hasta noviembre de 1996 se habían notificado 94997 casos de sida, con una incidencia acumulada de 68,1 por 100000 habitantes (1), y 3) el impacto que la epidemia tiene sobre el sistema de atención de la salud, fenómeno que ha exigido cada vez más esfuerzos de los poderes públicos para prevenir y controlar la dolencia. Ese escenario evidencia la necesidad de comprender las implicaciones de las diversas barreras que existen en el acceso a los servicios y de considerar los costos sociales y humanos de la infección por VIH, las necesidades y demandas de las personas infectadas y las consecuencias que puede tener para esas personas no ser atendidas con prontitud.

Al comienzo de la epidemia, el sida se consideraba una dolencia terminal y los costos de tratarla aumentaban solo en los últimos meses de vida de los pacientes. Los nuevos conocimientos sobre la infección por VIH y el sida han puesto a nuestra disposición un creciente arsenal diagnóstico y terapéutico que facilita la posibilidad de intervenir en estadios cada vez más tempranos de la evolución del síndrome. Esta situación, junto con el incremento del número de casos, ha generado una demanda sustancial de acciones y servicios de salud y los costos de tratamiento son cada vez más elevados. Por otro lado, nuevos factores de riesgo - entre ellos la pobreza, la poca escolaridad y la falta de acceso a servicios de salud- se vinculan no solo con el sida sino con episodios de violación de los derechos humanos, en el sentido de que los productos y servicios que deberían dedicarse a satisfacer las necesidades de las poblaciones de bajos ingresos no se están distribuyendo ni utilizando como es debido (2).

En el Brasil no son comunes los estudios que enfocan la utilización de los servicios, a pesar de que ese tipo de investigación es fundamental para evaluar aspectos como el acceso de las personas con infección por VIH al sistema de salud. Se sabe muy poco sobre los patrones de uso de las actividades y servicios por parte de los pacientes infectados por VIH y sobre los factores que podrían influir en ello (por ejemplo, las necesidades de los pacientes y de los servicios, el financiamiento de la atención que reciben y la discriminación social -aun por parte de los profesionales de salud-que resulta en la segregación y marginación de los pacientes seropositivos). Tampoco está claro hasta qué grado las barreras en el acceso a los servicios y actividades pueden influir en la progresión de la infección por VIH. Desde ese punto de vista, en este estudio nos propusimos evaluar la relación entre variables sociodemográficas, de comportamiento y, sobre todo, de dificultades en el uso de los servicios de salud y la evolución al sida en individuos afectados por VIH que acuden a los servicios públicos de salud a donde se remiten esos pacientes. El objetivo principal del estudio fue analizar la relación entre la utilización de los servicios de atención sanitaria y la progresión al sida entre pacientes diagnosticados de infección por VIH.

\section{MATERIALES Y MÉTODOS}

\section{Población}

Este estudio de tipo prospectivo no concurrente se desarrolló en la ciudad de Belo Horizonte del estado de Minas Gerais, Brasil, en tres servicios públicos de salud de referencia para pacientes con VIH y sida: 1) el servicio ambulatorio del Centro de Adiestramiento y Referencia Aids/DST Orestes Diniz (CAR) de la Prefectura Municipal de Belo Horizonte en convenio con la Universidad Federal de Minas Gerais (UFMG); 2) el Hospital Eduardo de Menezes (HEM) de la Fundación Hospitalaria del Estado de Minas Gerais, y 3) el Hospital de Clínicas de la UFMG (HCL).

La población de estudio se integró con todas las personas diagnosticadas de infección por VIH que fueron recibidas por primera vez en el CAR para evaluación y atención médica entre el 1 de enero de 1989 y el 31 de diciembre de 1992. El período de seguimiento para verificar el posible diagnóstico final de sida se extendió hasta el 31 de diciembre de 1994. Al término del período de estudio, los individuos seleccionados habían estado registra- dos en los servicios un mínimo de 2 años. Se verificó también el internamiento de participantes en el HEM o el HCL durante el mismo período, confrontando los datos de nacimiento.

Se seleccionaron para su estudio en este trabajo variables constantes derivadas de la revisión de registros médicos, psicológicos, sociales y de laboratorio en los expedientes clínicos de los participantes. El uso de ese tipo de datos por lo general implica limitaciones en cuanto a la calidad de la información obtenida. A veces la información buscada no se encuentra, pero en este estudio solamente $1,4 \%$ de los expedientes seleccionados se excluyeron del análisis por esa razón. Además, la información puede copiarse mal, lo cual en este trabajo se minimizó gracias a que: a) en los servicios de salud se había establecido prontamente la estandarización de procedimientos y registros relacionados con el sida, ya vigente en el período de estudio; b) los profesionales que atendían a los pacientes llevaban a cabo investigaciones frecuentes y, por lo tanto, apreciaban el registro correcto de datos, y c) los indicadores de uso de los servicios se escogieron de entre las experiencias más comunes de los pacientes y podían definirse con precisión.

Los datos pertinentes se anotaron en un formulario estandarizado que se había ensayado con anticipación. Todos los investigadores involucrados en el estudio recibieron adiestramiento para esa actividad de acuerdo con manuales de instrucción detallados. Después de la recolección de datos, se escogieron aleatoriamente $5 \%$ de los formularios llenados y la concordancia se evaluó mediante el índice kappa. De las 35 variables evaluadas, 29 mostraron una concordancia excelente (kappa $>0,75$ ) y las seis restantes, una concordancia de razonable a buena $(0,50<$ kappa $<0,74)$.

Se obtuvo información sobre las siguientes variables de exposición: 1) indicadores de dificultad al buscar atención de salud durante el transcurso de la estadía en los servicios estudiados: a) servicio utilizado, b) período transcurrido entre el momento de obtener el resultado preliminar de la 
prueba de VIH y la primera visita a los servicios, c) período transcurrido entre la primera y la segunda visitas a los servicios, d) período transcurrido entre la toma de muestras para el primer ELISA en laboratorio y la comunicación de los resultados al paciente, e) período transcurrido entre la primera visita a los servicios y la realización del primer recuento de linfocitos $T, f$ ) número promedio de consultas realizadas en un año, g) número de lapsos mayores de 6 meses entre dos consultas, h) registro de administración de azidovudina (AZT), i) número de días de internamiento hospitalario durante el período estudiado, y j) año civil de entrada en el servicio de salud; 2) indicadores de fuentes de infección o de comportamientos de riesgo: a) hemofilia, b) transfusión de sangre o hemodiálisis, c) relaciones sexuales con una persona diagnosticada de infección por $\mathrm{VIH}$, d) relaciones sexuales con hombres, e) relaciones sexuales con mujeres, f) número de compañeros sexuales durante la vida, g) haber sido trabajador sexual, $h$ ) frecuencia del uso de preservativo, i) uso de cualquier droga inyectable, j) uso compartido de jeringas; y 3) variables sociodemográficas: a) edad, b) sexo, c) color de la piel, d) estado civil, e) ciudad de residencia, f) escolaridad, $g$ ) ingresos individuales al mes, h) seguro de enfermedad. Toda la información sociodemográfica se obtuvo durante la primera visita de los pacientes a los servicios de salud. Las variables continuas se categorizaron de acuerdo con una distribución por cuartiles, considerándose la mediana como punto de corte.

Como variable de medición del resultado se usó el tiempo sin sida. Para los individuos que no desarrollaron el sida, esa variable se definió como el tiempo transcurrido entre la fecha de la clasificación clínica inicial y la fecha del diagnóstico de sida, si ambas estaban registradas en la historia clínica. Para los que no progresaron al sida, la variable se definió como el tiempo transcurrido entre la fecha de clasificación clínica inicial y la fecha de la última visita a los servicios durante el período estudiado. Entre estos, se observaron dos subgrupos: 1) las personas que siguie- ron el tratamiento y fueron monitoreadas hasta el final del período de estudio y 2) las personas que abandonaron el tratamiento y fueron monitoreadas solamente durante el período en que asistieron a los servicios.

La conversión al sida se monitoreó mediante la clasificación de los sujetos por estadio de enfermedad al emitirse el diagnóstico inicial y en la última visita a los servicios. Esa distribución se basó en las tres categorías clínicas (" $\mathrm{A}$ ", " $\mathrm{B}$ " $\mathrm{y}$ " $\mathrm{C}$ ") propuestas para adolescentes y adultos infectados por VIH en el sistema de clasificación de los Centros para el Control y la Prevención de Enfermedades (CDC) de los Estados Unidos de América (3). La categoría " $\mathrm{A}$ " se refiere a pacientes con infección por VIH asintomática o con infección aguda o linfadenopatía generalizada persistente. La categoría " $\mathrm{B}$ " corresponde a estadios clínicos sintomáticos no clasificables como " $\mathrm{A}$ " ni " $\mathrm{C}$ ". $\mathrm{La}$ categoría " $\mathrm{C}$ " comprende los estadios clínicos que indican progresión al sida. Esa progresión se evaluó únicamente en aquellos individuos que se clasificaron inicialmente en las categorías " $\mathrm{A}$ " $y$ " $\mathrm{B}$ ". Cabe aclarar que el recuento de linfocitos T CD4+ no pudo utilizarse para clasificar a los pacientes, ya que esa información falta en un número considerable de expedientes. Esto no refleja necesariamente lagunas o incorrecciones en el registro de los datos, sino más bien el hecho de que esa importante prueba no estaba disponible durante el período estudiado.

\section{Análisis estadístico}

Para determinar si las dificultades en la utilización de los servicios de salud y las características sociodemográficas de los pacientes constituyeron factores de riesgo en el deterioro de la salud, se realizó un análisis univariado en el cual se examinó la asociación entre cada variable seleccionada y la progresión al sida. Los datos categóricos se analizaron con la prueba de ji cuadrado $\left(\chi^{2}\right)$ y los continuos con la prueba $t$ de Student. El riesgo relativo de desarrollar sida se calculó con un intervalo de confianza de 95\% por medio del modelo de riesgo proporcional de Cox (4).

Al estimar la probabilidad acumulada del tiempo sin sida de los participantes, se utilizó el método de Kaplan-Meier (5). La comparación de diferentes curvas para los subgrupos correspondientes a las variables de exposición se basó en la prueba logrank. Se consideró estadísticamente significativo el valor de 0,05.

El efecto independiente de las variables seleccionadas en la progresión al sida se verificó mediante el modelo de riesgo proporcional de Cox. La modelación se hizo por eliminación secuencial no automática, empezando por las variables que en el análisis univariado presentaron una asociación estadística al nivel de $P<0,10$ o que se consideraban importantes desde el punto de vista clínico o epidemiológico. La prueba de Wald se usó al evaluar la importancia de cada variable y la prueba de la razón de máxima verosimilitud para comparar los modelos y seleccionar el más adecuado (6).

\section{RESULTADOS}

\section{Estadística descriptiva}

El estudio incluyó 1079 personas que habían tenido resultados positivos a las pruebas de VIH y fueron admitidas a los servicios ambulatorios del CAR entre el 1 de enero de 1989 y el 31 de diciembre de 1992. De ellas, 758 fueron clasificadas como casos en estadio "A" o "B", 279 habían sido diagnosticadas de sida al entrar en los servicios y las 42 restantes... Durante el período de seguimiento, 388 (36\%) fueron hospitalizadas en el HEM o el HCL. Las razones para su remisión al servicio ambulatorio fueron confirmación del diagnóstico en 37,4\%, un diagnóstico inicial en 25,8\% y seguimiento o tratamiento de $25,7 \%$. En 11,1\% se ignoró la razón. En 60,2\% de los casos, estos fueron encaminados al servicio por un profesional de salud. En el cuadro 1 se presentan las características sociodemográficas y de comportamiento o fuentes de infección de los participantes. 
CUADRO 1. Distribución de variables sociodemográficas y de comportamiento o fuente de infección entre pacientes con VIH atendidos en servicios públicos de salud. Belo Horizonte (Minas Gerais), Brasil, 1989-1994

\begin{tabular}{lrr}
\hline \multicolumn{1}{c}{ Variable } & No. $^{\mathrm{a}}$ & $\%$ \\
\hline Sociodemográfica & & \\
Edad (<30 años) & 1079 & 53,1 \\
Ser soltero & 1077 & 72,1 \\
Ser hombre & 1079 & 85,3 \\
Piel blanca & 1033 & 56,4 \\
Escolaridad ( $\geq 8$ años) & 1054 & 61,2 \\
Residente de Belo Horizonte & 1070 & 68,1 \\
<3 salarios mínimos mensuales & 985 & 59,5 \\
Cobertura por Seguridad Social & 894 & 86,6 \\
De comportamiento/Fuente de infección & & \\
Hemofilia & 1054 & 2,5 \\
Transfusión/Hemodiálisis & 1051 & 11,2 \\
Uso de una droga inyectable & 977 & 51,0 \\
Compartir jeringas & 1019 & 12,8 \\
Parejas sexuales (> 100) & 893 & 27,5 \\
Sexo con personas positivas a VIH & 701 & 34,1 \\
Antecedentes de prostitución personal & 912 & 9,8 \\
Antecedentes de ETS & 1055 & 73,0 \\
Uso de preservativos (<50\% de las veces) & 893 & 80,5 \\
Sexo exclusivamente con hombres & 1060 & 20,9 \\
Sexo con hombres y mujeres & 1060 & 38,2 \\
Sexo exclusivamente con mujeres & 1060 & 22,4 \\
Mujeres heterosexuales & 1060 & 13,6 \\
Práctica sexual ignorada o abstinencia & 1060 & 5,0 \\
\hline
\end{tabular}

a Los números varían debido a la falta de datos en los expedientes de pacientes.

${ }^{\mathrm{b}} \mathrm{ETS}=$ enfermedades de transmisión sexual.

CUADRO 2. Distribución de los pacientes con VIH atendidos en servicios públicos de salud, según su clasificación por estadio y progresión de la infección. Belo Horizonte (Minas Gerais), Brasil, 1989-1994

\begin{tabular}{ccccc}
\hline \multirow{2}{*}{$\begin{array}{c}\text { Clasificación } \\
\text { inicial" }\end{array}$} & \multicolumn{3}{c}{ Clasificación finala } & $\begin{array}{c}\text { Total } \\
\text { No. }(\%)\end{array}$ \\
\cline { 2 - 4 } "A" & "A" & "B" & "C" & $538(51,9)$ \\
"B" & $-C 44$ & 46 & 148 & $220(21,2)$ \\
"C" & - & 69 & 151 & $279(26,9)$ \\
Total: No. (\%) & $344(33,2)$ & $115(11,1)$ & $578(55,7)$ & $1037(100,0)$ \\
\hline
\end{tabular}

a "A" = Asintomático o con infección aguda o linfodenopatía generalizada persistente.

"B" = Sintomático, ni "A" ni "C".

"C" = Con enfermedades indicadoras del sida.

El cuadro 2 muestra la distribución de pacientes según su clasificación inicial y final por estadio de la enfermedad, lo que permite apreciar su progresión al sida. De los 758 pacientes clasificados como " $\mathrm{A}$ " o " $\mathrm{B}$ " al inicio, $299(39,5 \%)$ progresaron al sida (incidencia acumulada) mientras que 459
$(60,5 \%)$ no progresaron $(45,4 \%$ permanecieron en el estadio " $A$ "; $9,1 \%$ en el "B"; y 6,1\% evolucionaron de la categoría "A" a la "B"). El coeficiente de incidencia personas-tiempo para el sida fue de $25,4 \%$ personas-mes.

En lo que se refiere a las características seleccionadas para indicar dificul- tad en el uso de los servicios de salud, los resultados indicaron que, en el período inmediatamente anterior al diagnóstico de sida, el número promedio de consultas hechas por persona al año fue de 17,3 y la mediana, de 8,8 . El uso de AZT se registró en $333(31,9 \%)$ de las historias clínicas. Se habían remitido por lo menos una vez para hospitalización 494 pacientes $(46,9 \%)$ y $461(43,7 \%)$ habían sido internados una vez como mínimo. De los que habían sido internados, $296(64,2 \%)$ tuvieron su primera hospitalización en el HEM y $77(16,7 \%)$ en el HCL. Entre la primera visita y el primer regreso al servicio ambulatorio pasó una media de 50,1 días y una mediana de 20. Transcurrieron una media de 50,4 días y una mediana de 23 entre la toma de material para la primera prueba de laboratorio ELISA y la comunicación de los resultados al paciente. El lapso entre la comunicación al paciente de los resultados de la prueba para VIH y la primera visita tuvo una media de 150,6 días y una mediana de 47. Entre la primera visita al servicio y la prueba de recuento de linfocitos $T$ hubo un período que duró una media de 317,8 días y una mediana de 176.

\section{Análisis univariado}

En el cuadro 3 aparece la proporción de pacientes que desarrollaron sida entre los positivos a VIH clasificados inicialmente en las categorías " $\mathrm{A}$ " $\mathrm{y}$ " $\mathrm{B}$ ", el riesgo relativo $(R R)$ estimado por la función de riesgo instantáneo (hazard) y los intervalos de confianza de $95 \%$, según las características seleccionadas. El riesgo relativo de desarrollar sida en los pacientes mayores de 30 años fue 1,7 veces mayor que entre los más jóvenes. En los que tenían antecedentes de enfermedades de transmisión sexual (ETS), el riesgo relativo fue 1,5 veces mayor que entre los que nunca habían tenido esas enfermedades. Además, la incidencia acumulada de sida fue más alta entre pacientes con menos de 8 años de escolaridad, los que no estaban cubiertos por la seguridad social y los hemofíli- 
CUADRO 3. Análisis univariado de progresión al sida según ciertas características sociodemográficas de pacientes con infección por VIH atendidos en servicios públicos de salud. Belo Horizonte (Minas Gerais), Brasil, 1989-1994

\begin{tabular}{|c|c|c|c|c|}
\hline Variable & $\begin{array}{l}\text { Total }^{\text {a }} \\
\text { No. }\end{array}$ & $\begin{array}{l}\text { Sidab } \\
\text { No. (\%) }\end{array}$ & $\begin{array}{l}\text { Riesgo relativoc } \\
\text { (IC95\%) }\end{array}$ & Valor de $P$ \\
\hline $\begin{array}{l}\text { Edad } \\
\geq 30 \text { años } \\
<30 \text { años }\end{array}$ & $\begin{array}{l}357 \\
343\end{array}$ & $\begin{array}{l}178(49,9) \\
114(33,2)\end{array}$ & $\begin{array}{c}1,73(1,36 \text { a } 2,19)^{d} \\
1,0\end{array}$ & $<0,001$ \\
\hline $\begin{array}{l}\text { Sexo } \\
\text { Femenino } \\
\text { Masculino }\end{array}$ & $\begin{array}{l}120 \\
580\end{array}$ & $\begin{array}{r}46(38,3) \\
246(42,4)\end{array}$ & $\begin{array}{c}0,77(0,56 \text { a } 1,05) \\
1,0\end{array}$ & 0,096 \\
\hline $\begin{array}{l}\text { Color de la piel } \\
\text { Negra/mulata } \\
\text { Blanca }\end{array}$ & $\begin{array}{l}305 \\
372\end{array}$ & $\begin{array}{l}112(36,7) \\
171(46,0)\end{array}$ & $\begin{array}{c}0,75(0,59 \text { a } 0,96)^{d} \\
1,0\end{array}$ & 0,019 \\
\hline $\begin{array}{l}\text { Unión/Estado civil } \\
\text { Inestable } \\
\text { Estable }\end{array}$ & $\begin{array}{l}567 \\
131\end{array}$ & $\begin{array}{r}234(41,3) \\
57(43,5)\end{array}$ & $\begin{array}{c}0,85(0,63 \text { a } 1,13) \\
1,0\end{array}$ & 0,263 \\
\hline $\begin{array}{l}\text { Residencia } \\
\text { En otra ciudad } \\
\text { En Belo Horizonte }\end{array}$ & $\begin{array}{l}228 \\
468\end{array}$ & $\begin{array}{r}94(41,2) \\
197(42,1)\end{array}$ & $\begin{array}{c}1,03(0,80 \text { a } 1,31) \\
1,0\end{array}$ & 0,831 \\
\hline $\begin{array}{l}\text { Escolaridad } \\
<8 \text { años } \\
\geq 8 \text { años }\end{array}$ & $\begin{array}{l}268 \\
417\end{array}$ & $\begin{array}{l}118(44,0) \\
167(40,1)\end{array}$ & $\begin{array}{c}1,25(0,98 \text { a } 1,58) \\
1,0\end{array}$ & 0,068 \\
\hline $\begin{array}{l}\text { Ingresos personales al mes } \\
<3 \text { salarios mínimos } \\
\geq 3 \text { salarios mínimos }\end{array}$ & $\begin{array}{l}380 \\
272\end{array}$ & $\begin{array}{l}147(38,7) \\
124(45,6)\end{array}$ & $\begin{array}{c}0,85(0,67 \text { a } 1,08) \\
1,0\end{array}$ & 0,195 \\
\hline $\begin{array}{l}\text { Seguridad } \\
\text { No } \\
\text { Sí }\end{array}$ & $\begin{array}{r}72 \\
533\end{array}$ & $\begin{array}{r}34(47,2) \\
222(41,7)\end{array}$ & $\begin{array}{c}1,10(0,77 \text { a } 1,59) \\
1,0\end{array}$ & 0,598 \\
\hline $\begin{array}{l}\text { Número de parejas sexuales } \\
\geq 100 \\
<100\end{array}$ & $\begin{array}{l}153 \\
449\end{array}$ & $\begin{array}{r}59(38,6) \\
182(40,5)\end{array}$ & $\begin{array}{c}1,00(0,74 \text { a } 1,34) \\
1,0\end{array}$ & 0,980 \\
\hline $\begin{array}{l}\text { Comportamiento de riesgo } \\
\text { Hemofilia/Transfusión } \\
\text { Usuario de drogas inyectables } \\
\text { Sexual }\end{array}$ & $\begin{array}{r}45 \\
73 \\
561\end{array}$ & $\begin{array}{r}24(53,3) \\
29(39,7) \\
229(40,8)\end{array}$ & $\begin{array}{c}1,42(0,93 \text { a } 2,17) \\
1,07(0,73 \text { a } 1,58) \\
1,0\end{array}$ & $\begin{array}{l}0,102 \\
0,717\end{array}$ \\
\hline $\begin{array}{l}\text { Antecedentes de ETSe } \\
\text { Sí } \\
\text { No }\end{array}$ & $\begin{array}{l}504 \\
187\end{array}$ & $\begin{array}{r}228(45,2) \\
59(31,6)\end{array}$ & $\begin{array}{c}1,57(1,18 \text { a } 2,09)^{d} \\
1,0\end{array}$ & 002 \\
\hline
\end{tabular}

${ }^{a}$ Los totales varían debido a la falta de datos en los expedientes de pacientes.

${ }^{\text {b Número de pacientes que desarrollaron sida. }}$

c Obtenido con el cálculo hazard del modelo de Cox.

${ }^{\mathrm{d}}$ Asociación estadísticamente significativa $(\leq 0,05)$.

e ETS = enfermedades de transmisión sexual.
Sin embargo, no se detectó ninguna asociación estadísticamente significativa en esas variables.

En cuanto a los indicadores de dificultades relacionadas con los servicios de atención de salud (cuadro 4), la incidencia acumulada más alta de sida se observó entre los que habían tenido por lo menos un día de internamiento hospitalario. El riesgo de que esos pacientes progresaran al sida fue 1,5 veces mayor que en los que no tenían antecedentes de internamiento. Las personas a quienes se les hizo posible recibir AZT antes del diagnóstico de sida tuvieron una mayor incidencia acumulada de sida que las que no usaron ese medicamento, pero no hubo una diferencia estadísticamente significativa entre los dos grupos. Cabe notar la tendencia a disminución del riesgo que se observó en las otras variables que indican dificultad en el uso de los servicios. Así, el riesgo de desarrollar sida fue menor y estadísticamente significativo, en los pacientes que tuvieron menos de 8,8 consultas al año, lapsos mayores de 6 meses entre dos consultas, un lapso mayor de 20 días entre la primera visita y la segunda, un período superior a 47 días entre la prueba del VIH y la primera visita, y un período de más de 176 días entre la primera visita y el primer examen de linfocitos $\mathrm{T}$. También se observó menor riesgo de desarrollar sida, sin asociación estadísticamente significativa, entre los pacientes que tuvieron que esperar más de 24 días desde la toma del material para la prueba ELISA hasta que les comunicaron los resultados y entre los que fueron admitidos a los servicios en 1990 y 1991.

En cuanto a indicadores de progresión al sida, la incidencia acumulada de sida fue mayor entre los clasificados al inicio en la categoría "B". Esos pacientes tuvieron un riesgo 5,6 veces mayor de desarrollar sida que los que fueron clasificados en la categoría "A." Las personas con un primer recuento de linfocitos T CD4+ menor de $200 / \mathrm{mm}^{3}$ también tuvieron un riesgo más alto que las que tuvieron recuentos superiores a $200 / \mathrm{mm}^{3}$. 
CUADRO 4. Análisis univariado de progresión al sida de pacientes con infección por VIH atendidos en servicios públicos de salud, según indicadores de dificultad en el uso de esos servicios. Belo Horizonte (Minas Gerais), Brasil, 1989-1994

\begin{tabular}{|c|c|c|c|c|c|}
\hline Variable & & $\begin{array}{l}\text { Total } \\
\text { No. }\end{array}$ & $\begin{array}{l}\text { Sidab } \\
\text { No. (\%) }\end{array}$ & $\begin{array}{l}\text { Riesgo relativo } \\
\text { (IC95\%) }\end{array}$ & Valor de $P$ \\
\hline Consultas al año ${ }^{d}$ & $\begin{array}{l}<8,8 \\
\geq 8,8\end{array}$ & $\begin{array}{l}357 \\
343\end{array}$ & $\begin{array}{l}128(35,9) \\
164(47,8)\end{array}$ & $\begin{array}{c}0,24(0,19 \text { a } 0,30)^{e} \\
1,0\end{array}$ & $<0,001$ \\
\hline $\begin{array}{l}\text { Lapso }>6 \text { meses } \\
\text { entre dos consultas }\end{array}$ & $\begin{array}{l}\text { Sí } \\
\text { No }\end{array}$ & $\begin{array}{l}253 \\
447\end{array}$ & $\begin{array}{r}89(35,2) \\
203(45,4)\end{array}$ & $\begin{array}{c}0,23(0,18 \text { a } 0,30)^{e} \\
1,0\end{array}$ & $<0,001$ \\
\hline Uso de azidovudina & $\begin{array}{l}\text { No } \\
\text { Sí }\end{array}$ & $\begin{array}{l}534 \\
122\end{array}$ & $\begin{array}{r}183(34,3) \\
65(53,3)\end{array}$ & $\begin{array}{c}1,03(0,77 \text { a } 1,36) \\
1,0\end{array}$ & 0,860 \\
\hline Días de hospitalización & $\begin{array}{c}\geq 1 \\
\text { Ninguno }\end{array}$ & $\begin{array}{r}79 \\
612\end{array}$ & $\begin{array}{r}55(69,6) \\
233(38,1)\end{array}$ & $\begin{array}{c}1,49(1,11 \text { a } 1,99)^{\mathrm{e}} \\
1,0\end{array}$ & 0,009 \\
\hline $\begin{array}{l}\text { Lapso entre primera } \\
\text { y segunda visitas }\end{array}$ & $\begin{array}{l}>20 \text { días } \\
\leq 20 \text { días }\end{array}$ & $\begin{array}{l}401 \\
269\end{array}$ & $\begin{array}{l}147(36,7) \\
124(46,1)\end{array}$ & $\begin{array}{c}0,75(0,59 \text { a } 0,95)^{\mathrm{e}} \\
1,0\end{array}$ & 0,018 \\
\hline $\begin{array}{l}\text { Lapso entre toma } \\
\text { de muestra y } \\
\text { comunicación de } \\
\text { resultados (ELISA) }\end{array}$ & $\begin{array}{l}>23 \text { días } \\
\leq 23 \text { días }\end{array}$ & $\begin{array}{l}215 \\
175\end{array}$ & $\begin{array}{l}69(32,1) \\
75(42,9)\end{array}$ & $\begin{array}{c}0,77(0,55 \text { a } 1,07) \\
1,0\end{array}$ & 0,120 \\
\hline $\begin{array}{l}\text { Lapso entre prueba de } \\
\text { detección de VIH y } \\
\text { primera visita }\end{array}$ & $\begin{array}{l}>47 \text { días } \\
\leq 47 \text { días }\end{array}$ & $\begin{array}{l}194 \\
120\end{array}$ & $\begin{array}{l}74(38,1) \\
65(54,2)\end{array}$ & $\begin{array}{c}0,68(0,48 \text { a } 0,95)^{e} \\
1,0\end{array}$ & 0,022 \\
\hline $\begin{array}{l}\text { Lapso entre primera } \\
\text { visita y recuento de } \\
\text { linfocitos } T\end{array}$ & $\begin{array}{l}>176 \text { días } \\
\leq 176 \text { días }\end{array}$ & $\begin{array}{r}88 \\
221\end{array}$ & $\begin{array}{r}35(39,8) \\
104(47,1)\end{array}$ & $\begin{array}{c}0,44(0,30 \text { a } 0,66)^{e} \\
1,0\end{array}$ & $<0,001$ \\
\hline $\begin{array}{l}\text { Año de entrada al servicio } \\
\text { de salud }\end{array}$ & $\begin{array}{l}1989 \\
1990 \\
1991 \\
1992\end{array}$ & $\begin{array}{l}123 \\
176 \\
176 \\
225\end{array}$ & $\begin{array}{l}53(43,1) \\
86(48,9) \\
70(39,8) \\
83(36,9)\end{array}$ & $\begin{array}{c}1,00(0,72 \text { a } 1,37) \\
0,95(0,69 \text { a } 1,29) \\
0,74(0,51 \text { a } 1,07) \\
1,0\end{array}$ & $\begin{array}{l}0,980 \\
0,732 \\
0,106\end{array}$ \\
\hline $\begin{array}{l}\text { Clasificación inicial por } \\
\text { estadio }^{\dagger}\end{array}$ & $\begin{array}{l}\text { "B" } \\
\text { "A" }\end{array}$ & $\begin{array}{l}198 \\
502\end{array}$ & $\begin{array}{l}147(74,2) \\
145(28,9)\end{array}$ & $\begin{array}{c}5,68(4,48 \text { a } 7,21)^{\mathrm{e}} \\
1,0\end{array}$ & $<0,001$ \\
\hline $\begin{array}{l}\text { Linfocitos T CD4+ en el } \\
\text { primer recuento }\end{array}$ & $\begin{array}{l}<200 / \mathrm{mm}^{3} \\
\geq 200 / \mathrm{mm}^{3}\end{array}$ & $\begin{array}{r}98 \\
217\end{array}$ & $\begin{array}{l}66(67,4) \\
77(35,5)\end{array}$ & $\begin{array}{c}2,66(1,91 \text { a } 3,72)^{\mathrm{e}} \\
1,0\end{array}$ & $<0,001$ \\
\hline
\end{tabular}

a Los totales varían debido a la falta de datos en los expedientes de pacientes.

b Número de pacientes que progresaron al sida.

c Obtenido con el cálculo hazard del modelo de Cox.

d Número medio de consultas al año, antes del diagnóstico de sida.

e Asociación estadísticamente significativa $(\leq 0,05)$.

f "A" = asintomático o con infección aguda o linfodenopatía generalizada persistente;

"B" = sintomático, ni " $\mathrm{A}$ " ni " $\mathrm{C}$ "; $\mathrm{y}$ "C" = con enfermedades indicadoras del sida.

\section{Período sin sida}

La mediana de tiempo sin sida fue de 32,4 meses (44,8 meses para los pacientes de categoría " $\mathrm{A}$ " y 4,1 meses para los de categoría "B"). Entre los de la categoría "A", las medianas de tiempo sin sida fueron significativamente mayores $(P<0,05)$ en aquellos que: tenían menos de 30 años (50,8 frente a 43,8); eran de piel negra o mulata (50,1 frente a 43,4); tuvieron menos de 8,8 consultas médicas al año $(48,1$ frente a 22,2$)$; experimentaron intervalos mayores de 6 meses entre dos consultas (50,1 frente a 28,1); no fueron hospitalizados (47,3 frente a $29,9)$; tuvieron un lapso > 177 días entre la primera visita y el primer recuento de linfocitos $\mathrm{T}(50,1$ frente a 44,2$)$; $y$ un recuento de linfocitos T CD4+ $\geq 200 / \mathrm{mm}^{3}(50,1$ frente a 28,1$)$. Los pacientes que ingresaron en el servicio de 1992 en adelante vivieron menos tiempo sin sida $(32,8$ frente a 56,5$)$

Dentro de la categoría "B", las medianas de tiempo sin sida, en meses, fueron significativamente mayores $(P$
$<0,05)$ para los pacientes que: tenían ingresos mensuales inferiores a tres salarios mínimos $(4,7$ frente a 3,8$)$; tuvieron menos de 8,8 consultas médicas al año (18,0 frente a 2,3$)$, tuvieron lapsos mayores de 6 meses entre dos consultas $(29,6$ frente a 3,0$)$; recibieron AZT (14,5 frente a 3,9); tuvieron un lapso $>20$ días entre la primera y la segunda visita $(7,5$ frente a 2,9$)$; y tuvieron un período $>23$ días entre la toma de material para la prueba ELISA y el conocimiento de los resultados $(10,9$ frente a 2,3). Las figuras 1 y 2 muestran curvas de Kaplan-Meier para toda la población, de acuerdo con el promedio anual de consultas y el número de días de internamiento, respectivamente.

\section{Análisis multivariado}

El efecto independiente de variables seleccionadas sobre el tiempo sin sida fue evaluado por medio del análisis de riesgo proporcional de Cox. Sobre la base de un análisis univariado (inclusión de variables con $P<0,10$ ), en las estimaciones de tiempo sin sida y en las consideraciones biológicas y epidemiológicas, se escogieron las siguientes variables para integrar un modelo: 1) promedio de consultas al año; 2) clasificación inicial según el estadio de la enfermedad; 3) lapsos mayores de 6 meses entre consultas; 4) uso de AZT; 5) color de la piel; 6) número de días de internamiento hospitalario; 7) tiempo entre la primera y segunda visitas; 8) edad en el momento de admisión en el servicio; 9) años de escolaridad; 10) antecedentes de enfermedades de transmisión sexual; y 11) sexo. Seguidamente se evaluó por eliminación secuencial, paso a paso y no automática, la importancia de esas variables.

Se excluyeron del análisis las siguientes variables, debido al gran número de valores para los cuales se carecía de información: primer recuento de linfocitos T CD4+; intervalo entre la prueba anterior de VIH y la primera visita; y el período entre la primera visita y el primer examen de linfocitos T. En el análisis univariado, todos los subgrupos correspondientes 
FIGURA 1. Tiempo sin sida (método Kaplan-Meier) de acuerdo con el número medio anual de consultas $(<8,8$ e $\geq 8,8)$ hechas por pacientes con infección por VIH atendidos en los servicios públicos de salud. Belo Horizonte (Minas Gerais), Brasil, 1989-1994

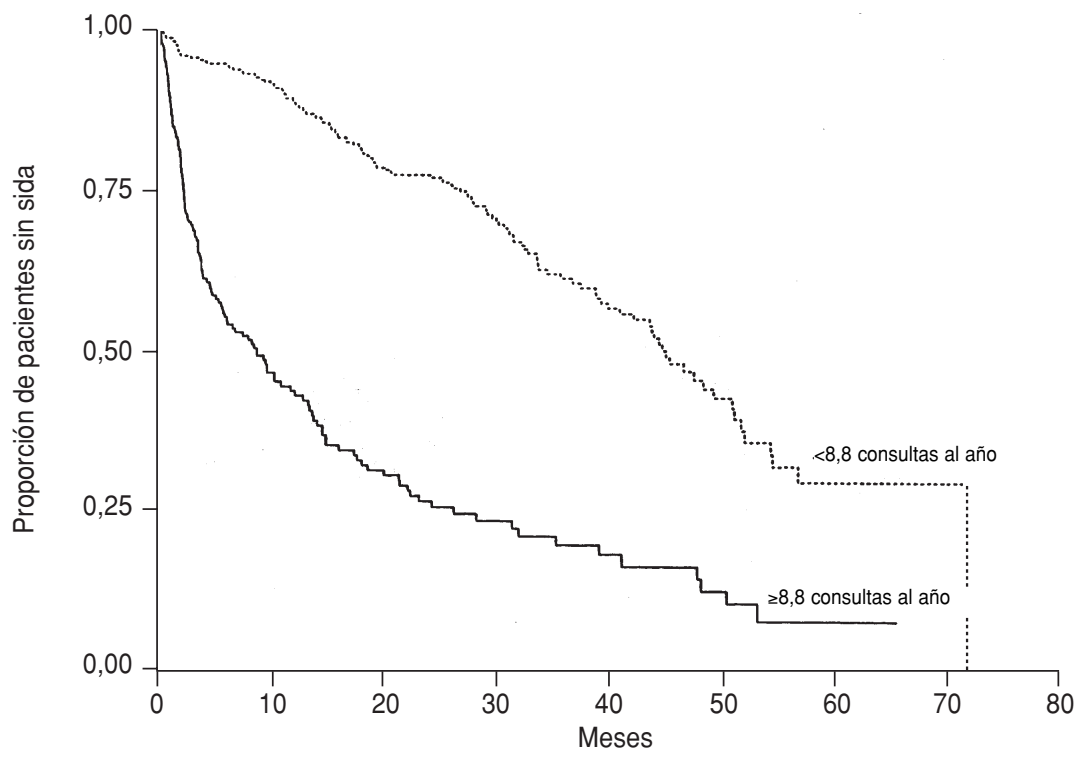

FIGURA 2. Tiempo sin sida (método Kaplan-Meier) de acuerdo con el número de días de hospitalización (ninguno $0 \geq 1$ día) de pacientes con infección por VIH atendidos en los servicios públicos de salud. Belo Horizonte (Minas Gerais), Brasil, 1989-1994

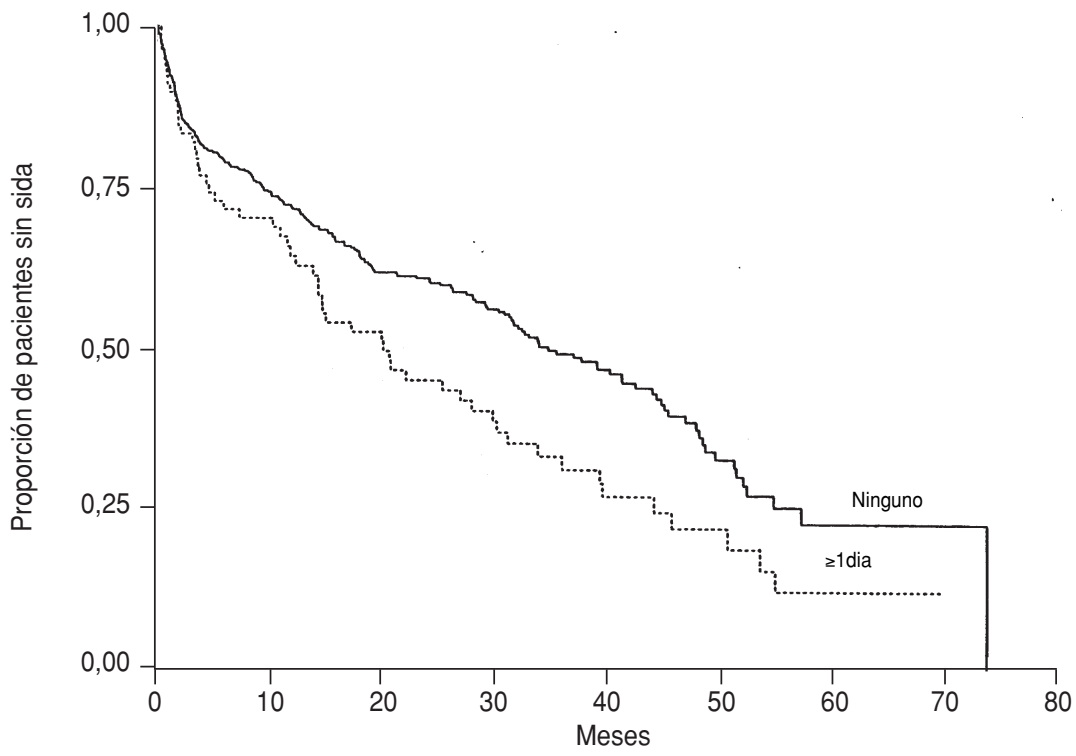

a esas variables tuvieron diferencias estadísticamente significativas.

En el cuadro 5 aparece el modelo final con los riesgos relativos estimados e intervalos de confianza de $95 \%$. La probabilidad de desarrollar sida fue menor en las personas que tuvieron menos de 8,8 consultas al año y, como mínimo, un intervalo mayor de 6 meses entre consultas. El mayor riesgo de progresar al sida correspondió a los pacientes que inicialmente fueron clasificados en el estadio " $\mathrm{B}$ ", los que no usaron AZT y los de 30 o más años de edad.
Variables importantes desde el punto de vista epidemiológico, como el color de la piel, los antecedentes de ETS y los de internamiento hospitalario, una vez ajustadas, no sostuvieron la asociación inicialmente detectada. El modelo se evaluó como adecuado mediante la prueba de la razón de máxima verosimilitud $\left(\chi^{2}=2,20 ; P=0,138\right)$.

\section{DISCUSIÓN}

Considerando cuán corto fue el período de seguimiento, la incidencia acumulada de sida en esta cohorte prevalente $^{3}$ fue relativamente alta $(39,5 \%)$ cuando se comparó con otros estudios en los que hubo períodos de seguimiento más largos (7-12). En parte, ello se debe a que se desconoce cuánto duró el período desde el inicio de la infección por VIH hasta la entrada en el servicio ambulatorio. No se sabe, por ejemplo, cuánto tiempo había estado cada individuo en los estadios clasificados como "A" y "B". Es posible que la entrada en el servicio fuera ya tardía en relación con la seroconversión por VIH. Para corregir esta falta de información, por lo menos en parte, en el análisis multivariado las variables se ajustaron según los estadios iniciales y el análisis temporal comenzó con la fecha del diagnóstico inicial registrado para clasificación en estadio " $\mathrm{A}$ " o " $\mathrm{B}$ ".

La mediana general de tiempo sin sida fue de 32,4 meses, menor que las que se encuentran en otros estudios (8-10), lo que no debe sorprender, por tratarse de una cohorte de prevalencia (9); la mediana se calculó a partir de la entrada de los pacientes en el servicio ambulatorio. El modelo final muestra que el mayor riesgo correspondió a los pacientes que entraron ya en estadio "B" (proceso clínico de infección más avanzado) y evolucionaron al sida, comparados con los de estadio " $\mathrm{A}$ " $(R R=4,83)$. De las variables sociodemográficas y de comportamiento, solo

\footnotetext{
3 Incluye solamente a personas ya diagnosticadas de la enfermedad que interesa; en este caso, de infección por VIH.
} 
CUADRO 5. Riesgo relativo (RR) estimado e intervalo de confianza de $95 \%$ (IC95\%) según el modelo final de análisis proporcional de Cox. Belo Horizonte (Minas Gerais), Brasil, 1989-1994

\begin{tabular}{ll}
\hline \multicolumn{1}{c}{ Variable } & $R R(I C 95 \%)$ \\
\hline Consultas al añoa $(<8,8)$ & $0,36(0,26$ a 0,50$)$ \\
Clasificación inicial por estudio (categoría "B") & $4,83(3,59$ a 6,48$)$ \\
Lapso >6 meses entre dos consultas (sí) & $0,37(0,25$ a 0,55$)$ \\
Uso de azidovudina (no) & $1,91(1,37$ a 2,64$)$ \\
Edad ( $\geq 30$ años) & $1,37(1,03$ a 1,84$)$ \\
\hline a Número medio de consultas al año, antes del diagnóstico de sida. \\
b "A" = asintomático o con infección aguda o linfodenopatía generalizada persistente; "B" = \\
sintomático, ni "A" ni "C"; "C" = con enfermedades indicadoras del sida.
\end{tabular}

la edad mostró una asociación independiente con la progresión al sida, después de ajustada para el análisis multivariado. Los pacientes cuya edad era $\geq 30$ años estaban en mayor riesgo de desarrollar el síndrome $(R R=1,37)$. Ese resultado concuerda con los hallazgos de Chaisson et al. (11), quienes en su estudio relatan que en los participantes mayores de edad el riesgo de progresar al sida aumentaba con cada año de edad adicional. Por otro lado, Brettle et al. (12) no observaron ninguna diferencia asociada con la edad en la progresión al sida.

Al enfocar aspectos relacionados con dificultades en el uso de los servicios de salud, este estudio mostró que la mediana del tiempo sin sida de pacientes clasificados en el estadio "B" fue significativamente mayor entre los que usaron AZT. En el análisis multivariado, el riesgo de tener peores resultados fue mayor en los que no usaron AZT que en los que sí la usaron. Es importante aclarar que los resultados de esta investigación reflejan la situación de los pacientes y las opciones de atención de salud disponibles en el período de 1989 a 1994. Así, en la población estudiada, solo cuatro personas señalaron que habían usado AZT antes de diciembre de 1990, y tres de ellas eran sintomáticas. Esos datos coinciden con la política de distribución de AZT por el sistema público de atención en ese período, que solo proporcionaba el medicamento a los casos sintomáticos de mayor gravedad. El medicamento solo se empezó a distribuir regular- mente a partir de 1994. Si bien algunos estudios indican los efectos beneficiosos del uso de AZT contra la progresión al sida (13-16), en otros trabajos no se corroboran esos resultados (17-19). Todavía persiste la polémica sobre si el tratamiento antivírico precoz produce efectos de largo plazo $(20,21)$, pero la monoterapia con AZT ya no se recomienda como tratamiento estándar inicial para la infección por VIH (22).

En relación con la dificultad de utilizar los servicios de salud, se observó una tendencia uniforme de todos los indicadores constituidos por lapsos temporales: los pacientes expuestos a períodos más largos estuvieron más tiempo sin sida. La misma tendencia se observó en otros indicadores de utilización de los servicios de salud. Los pacientes que tuvieron un menor número medio de consultas al año y aquellos que tuvieron por lo menos un intervalo mayor de 6 meses entre dos consultas también vivieron más tiempo sin sida. Después de ajustarse, estas dos últimas variables mantuvieron una asociación independiente con la progresión al sida.

Debido a la dificultad de obtener en los expedientes otros indicadores no relacionados con la utilización de los servicios de salud, el estudio se diseñó suponiendo que la menor utilización (menor número de consultas, mayores intervalos entre indicadores de utilización) expresa mayor dificultad de acceso a los servicios. Aday et al. (23) consideran que la frecuencia real de utilización del sistema es una medida que puede emplearse para comprobar el valor predictivo de los indicadores de acceso relacionados con el sistema y con las personas. Sin embargo, este estudio mostró que los indicadores de utilización no eran suficientes para enfocar de forma comprensiva la dificultad de acceso a los servicios. La utilización, como componente del acceso, expresa el final de un proceso de búsqueda y obtención de atención y, por lo tanto, hay diversos elementos que deben ser considerados cuando se analiza el acceso a los servicios, por ejemplo, el deseo de atención, la localización de los servicios y otros. De manera que el enfoque debe adaptarse a la luz de los resultados obtenidos.

Los pacientes con peores resultados de salud tienden a utilizar más los servicios y a utilizar los más complejos. Para una explicación posible de esta asociación hay que entender el acceso como un grado de ajuste entre las características de los servicios de salud y las de la población (24). Ese ajuste expresa, por un lado, la relación entre un conjunto de obstáculos para la búsqueda y obtención de atención (resistencia) y, por otro lado, la capacidad de la población para superarlos (poder de utilización) (25). De este modo, las personas que perciben el empeoramiento de su enfermedad y de los síntomas correspondientes pueden proyectar un mayor poder de utilización en la medida en que están más motivados para obtener la atención que necesitan y, en consecuencia, son más tolerantes con los obstáculos que enfrentan (por ejemplo, el tiempo de espera, la pérdida de turno, etc.). Además, la organización de los servicios puede ser más permeable al acceso de pacientes más graves, ofreciéndoles mecanismos facilitadores aun cuando haya pocos recursos. De hecho, la asociación entre el uso incrementado de la atención de salud y la mayor gravedad de la enfermedad atendida se ha notificado en varios estudios (26-29), lo cual refuerza la vigencia de los temas y resultados discutidos en este estudio.

Los resultados son útiles en la planificación y organización de los servicios de atención para personas con VIH 
porque permiten comprender la dinámica involucrada en la oferta de cuidados de salud y la utilización de los servicios por esa clientela. En las circunstancias en que una epidemia se propaga, con aumento de la incidencia en poblaciones de bajos ingresos, incremento de los costos de tratamiento y crisis en el financiamiento del sector de la salud, el acceso a las acciones y los servicios puede ser limitado por restricciones importantes, en particular para la clientela que usa los servicios públicos. Desde esa perspectiva y con objeto de beneficiar a las personas infectadas por VIH, los sistemas y servicios de salud deben vencer algunos obstáculos para asegurar el acceso a la atención necesaria, buscando incrementar la disponibilidad, equidad, integralidad y calidad de las acciones. Aparte de ello, debe darse prioridad a la intervención temprana con énfasis en la atención ambulatoria y su continuidad. En el área de la producción del conocimiento, convendría realizar estudios que incorporaran métodos cualitativos y trabajaran con fuentes primarias de información, para explorar más ampliamente el proceso de búsqueda y obtención de cuidados de salud por las personas positivas a VIH.

Agradecimiento. Este estudio fue financiado por la Organización Panamericana de la Salud, por conducto del Programa de Investigación y Desarrollo Tecnológico en Salud (Registro: HDP / HDR / HPC-HCA / RG / BRA ID 1013).

\section{REFERENCIAS}

1. Brasil, Ministerio de Salud, Programa Nacional de Enfermedades de Transmisión Sexual/Sida. AIDS Bol Epidemiol 1996;4:12.

2. Fontes M. Vulnerabilidade. Boletim de Direitos Humanos em HIV/Aids 1997;1:8-9.

3. USA, Centers for Disease Control and Prevention. 1993 Revised classification system for HIV infection and expanded surveillance case definition for AIDS among adolescents and adults. MMWR 1992;41:1-4

4. Cox DR, Oakes D. Analysis of survival data. New York: Chapman and Hall; 1984.

5. Kahn HA, Sempo CT. Statistical methods in epidemiology. 2a ed. New York: Oxford University Press; 1989:185-198.

6. Statistics and Epidemiology Research Corporation. EGRET. Seattle: SERC; 1993.

7. Goedert JJ, Landesman SH, Eyster ME, Biggar RJ. AIDS incidence in pregnant women, their babies, homosexual men, and hemophiliacs [abstract]. En: III International Conference on AIDS, Washington, DC, 1-5 June 1987. (Book of abstracts, abstract TP56, p. 71).

8. Guimarães MDC. Heterosexual transmission of the human immunodeficiency virus in Brazil: A comparison of sexual practices [Disertación de Doctorado en Ciencias]. Baltimore: Johns Hopkins University; 1992.

9. Alcabes P, Muñoz A, Vlahov D, Friedland GH. Incubation period of human immunodeficiency virus. Epidemiol Rev 1993;15:303-318.

10. Jacobson LP, Kirby AJ, Polk S, Phair JP, Besley $\mathrm{DR}$, Saah AJ, et al. Changes in survival after acquired immunodeficiency syndrome (AIDS): 1984-1991. Am J Epidemiol 1993;136:952-964.

11. Chaisson RE, Keruly JC, Moore RD. Race, sex, drug use, and progression of human immunodeficiency virus disease. $N$ Engl J Med 1995; 333:751-756.

12. Brettle RP, Mcneil AJ, Burns S, Gore SM, Bird AG, Yap PL, et al. Progression of HIV: Followup of Edinburgh injecting drug users with narrow seroconversion intervals in 19831985. AIDS 1996;10:419-430.
13. Volberding PA, Lagakos SW, Koch MA, Pettinelli C, Myers MW, Booth DK, et al. Zidovudine in asymptomatic human immunodeficiency virus infection: A controlled trial in persons with fewer than 500 CD4-positive cells per cubic millimeter. N Engl J Med 1990; 322:941-949.

14. Vella S, Giuliano M, Daily LG, Agresti MG, Giuliano M, Daily LG, et al. Long-term follow-up of zidovudine therapy in asymptomatic HIV infection: Results of a multicenter cohort study. I Acquir Immune Defic Syndr 1994;7:31-38.

15. Kinloch-de-Loës S, Hirschel BJ, Hoen B, Cooper DA, Tindall B, Carr A, et al. A controlled trial of zidovudine in primary human immunodeficiency virus infection. $N$ Engl J Med 1995;333:408-413.

16. Simberkoff MS, Hartigan PM, Hamilton JD, Day PL, Diamond GR, Dickinson GM, et al. Long-term follow-up of symptomatic HIVinfected patients originally randomized to early versus later zidovudine treatment: Report of a veterans affairs cooperative study. $J$ Acquir Immune Defic Syndr Hum Retrovirol 1996;11:142-150.

17. German AIDS Study Group. Survival of patients receiving zidovudine before or after AIDS diagnosis: Results of a German multicenter study. Clin Investig (Berlin) 1994;72: 111-116.

18. Concorde Coordinating Committee. Concorde:MCR/ANRS randomised double-blind controlled trial of immediate and deferred zidovudine in symptom-free HIV infection. Lancet 1994;343:871-881.

19. Volberding PA, Lagakos SW, Grimes JM, Stein DS, Rooney J, Meng TC, et al. A comparison of immediate with deferred zidovudine therapy for asymptomatic HIV-infected adults with CD4 cell counts of 500 or more per cubic millimeter. N Engl J Med 1995;333: 450-451.

20. Ho DD. Time to hit HIV, early and hard. N Engl J Med 1995;333:450-451.
21. Nadler JP. Early initiation of antiretroviral therapy for infection with human immunodeficiency virus: Considerations in 1996. Clin Infect Dis 1996;23:227-230.

22. Corey L, Holmes KK. Therapy for human immunodeficiency virus infection: What have we learned? [Editorial]. N Engl J Med 1996; 333:1142-1143.

23. Aday LA, Andersen R. A framework for the study of access to medical care. Health Serv Res 1974;9:208-220.

24. Donabedian A. Aspects of medical care administration: Specifying requirements for health care. Cambridge: Harvard University Press; 1973.

25. Frenk J. El concepto y la medición de accesibilidad. Salud Publica Mex 1985;27:438-453.

26. Solomon L, Frank R, Vlahov D, Astemborski J. Utilization of health services in a cohort of intravenous drug users with known HIV-1 serostatus. Am J Public Health 1992;81: 1285-1290.

27. Mor V, Fleishman JA, Dresser M, Piette MS. Variation in health service use among HIVinfected patients. Med Care 1992;30:17-29.

28. Zucconi SL, Jacobson LP, Schragger LK, Kass NE, Lave JR, Carson CA, et al. Impact of immunosuppression on health care use by men in the Multicenter AIDS Cohort Study. J Acquir Immune Defic Syndr 1994;7:607-616.

29. Nageswaran A, Kinghorn GR, Sshen RN Priestley CJ, Kyi TT. Hospital service utilization by HIV/AIDS patients and their management cost in a provisional genitourinary medicine department. Int J STD AIDS 1995;6: 336-344.

Manuscrito recibido el 25 de abril de 1997 y aceptado para publicación en versión revisada el 7 de abril de 1998. 
ABSTRACT A nonconcurrent prospective study was done on the relationship between a number of variables and the progression to AIDS among persons diagnosed with the human immunodeficiency virus (HIV). The variables included sociodemographic ones, behav-

\section{Use of health services and the progression to AIDS among HIV-infected persons in Belo Horizonte (Minas Gerais), Brazil}

ioral ones, and, most importantly, those persons' difficulties in obtaining public health services for HIV/AIDS. The course of the infection was monitored from the first to the last visit to the health services by means of an individual, diagnostic-based classification, using categories established in 1993 by the Centers for Disease Control and Prevention of the United States of America. Participating in the study were 758 patients seen between 1989 and 1992 in the public AIDS referral services of the city of Belo Horizonte (Minas Gerais). All the persons had been diagnosed with HIV and classified in a pre-AIDS stage. Both the patients who developed AIDS during the study as well as those who did not were assessed according to the selected study characteristics.

During the study period, $39.5 \%$ of the patients developed AIDS. For the group as a whole, the median time without AIDS was 32.4 months. Multivariate analysis showed that the patients who had less risk of developing AIDS were those who had had fewer than 8.8 medical consultations per year (relative risk $=0.36 ; 95 \%$ confidence interval, 0.26 to 0.50$)$ and an interval of at least 6 months between consultations $(R R=0.37 ; 95 \%$ $\mathrm{CI}, 0.25$ to 0.55$)$. The risk was greater in patients age 30 and older $(R R=1.37 ; 95 \% C I$, 1.03 to 1.84$)$, in those who were not treated with zidovudine (AZT) $(R R=1.91 ; 95 \%$ $\mathrm{CI}, 1.37$ to 2.64 ), and those who were initially classified in stage " $\mathrm{B}$ " of the disease $(R R=4.83 ; 95 \% \mathrm{CI}, 3.59$ to 6.48$)$. The results of this investigation show the dynamics of the supply and demand of services by these patients, and the information will be useful in planning and organizing care for persons with HIV. Recommendations include giving priority to early intervention with a focus on ongoing outpatient care, and more study of the process that persons with HIV follow in seeking and obtaining health care. 